\title{
An Atypical Case of Bartonella henselae Osteomyelitis and Hepatic Disease
}

\author{
Dionna M. Mathews, ${ }^{1}$ Katie M. Vance $\left(D,{ }^{2}\right.$ Pamela M. McMahon, ${ }^{2}$ Catherine Boston, ${ }^{3}$ \\ and Michael T. Bolton $\mathbb{1}^{4}$ \\ ${ }^{1}$ Our Lady of the Lake Children's Hospital, Baton Rouge, LA, USA \\ ${ }^{2}$ Division of Academic Affairs, Our Lady of the Lake Regional Medical Center, Baton Rouge, LA, USA \\ ${ }^{3}$ Pediatric Hematology/Oncology, Our Lady of the Lake Children's Hospital/St. Jude Affiliate Baton Rouge, Baton Rouge, LA, USA \\ ${ }^{4}$ Pediatric Infectious Diseases, Our Lady of the Lake Children's Hospital, Baton Rouge, LA, USA
}

Correspondence should be addressed to Michael T. Bolton; michael.bolton@fmolhs.org

Received 19 October 2017; Revised 3 March 2018; Accepted 22 March 2018; Published 5 April 2018

Academic Editor: Albert M. Li

Copyright ( $\odot 2018$ Dionna M. Mathews et al. This is an open access article distributed under the Creative Commons Attribution License, which permits unrestricted use, distribution, and reproduction in any medium, provided the original work is properly cited.

\begin{abstract}
Bartonella henselae is a Gram-negative bacterium and the causative agent of cat scratch disease (CSD). Atypical presentations of $B$. henselae that involve the musculoskeletal, hepatosplenic, cardiac, or neurologic systems are rare. In this case report, we describe a case of $B$. henselae osteomyelitis involving bilateral iliac bones complicated by hepatic lesions in a 12-year-old immunocompetent female patient. Although $B$. henselae is a rare cause of osteomyelitis, it should be considered when patients who present with fever, pain, and lymphadenopathy do not respond to routine osteomyelitis therapy.
\end{abstract}

\section{Introduction}

Bartonella henselae, a Gram-negative bacterium, is the causative agent of cat scratch disease (CSD) that typically involves the mononuclear phagocyte system and presents as local lymphadenopathy, often accompanied by fever [1]. $B$. henselae infections are thought to occur when a human is bitten or scratched by an infected cat [2-4] and may be transmitted by cat fleas or by an infected cat licking the nonintact skin of a human [5-7]. IgM is often elevated only briefly and is commonly normal during the course of the disease [8]. IgG titers greater than $1: 256$ are typically indicative of previous or active disease [6]. Children and teenagers make up approximately $80 \%$ of patients diagnosed with CSD [2]. Although infrequent, B. henselae can affect almost every organ system after hematogenous, lymphatic, or contiguous spread.

Bartonella osteomyelitis most frequently occurs in the spine. The pelvic girdle is the most common site of Bartonella osteomyelitis outside of the spine and occurs in $42 \%$ of all nonspinal cases [3]. Bartonella osteomyelitis typically presents as tenderness or pain in the affected area [4]. Magnetic resonance imaging (MRI) or radionuclide bone scanning is often used to diagnose osteomyelitis. Clinicians must rely on serologic testing, polymerase chain reaction testing (PCR), or Warthin-Starry silver staining to identify $B$. henselae as the causative organism because it does not grow in culture $[3,4]$. We present a case of $B$. henselae osteomyelitis involving bilateral iliac bones complicated by hepatic lesions in an immunocompetent patient.

\section{Case Presentation}

A previously healthy 12-year-old female presented to the emergency department 4 days after completing a 3-day course of trimethoprim/sulfamethoxazole prescribed for a urinary tract infection. She complained of a 9-day history of fever and 3-day history of left hip pain associated with joint movement. The patient denied trauma, erythema, or swelling in the area of pain, but exposure history uncovered prolonged cat contact. Her initial exam revealed full range of motion of all extremities though she exhibited tenderness to 


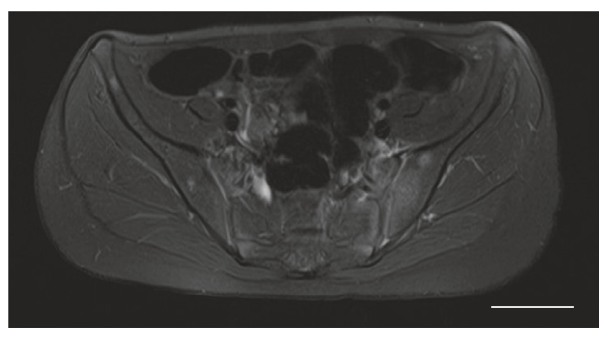

(a)

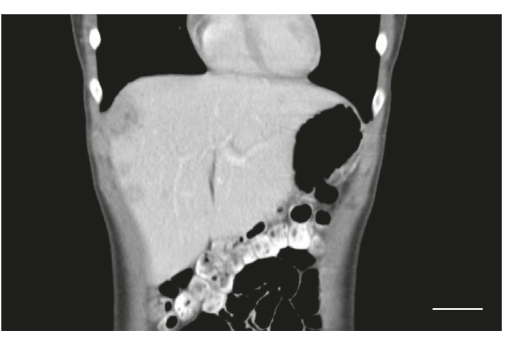

(b)

FIGURE 1: MRI and CT of patient's Bartonella bone and hepatic lesions. (a) An MRI obtained of the patient's pelvis shows small, scattered lesions throughout the iliac architecture. (b) A CT of the patient's abdomen shows hepatic lesions indicative of $B$. henselae. Bar, $5 \mathrm{~cm}$.

palpation of her left hip. The remainder of the exam was unremarkable, including absence of any lymphadenopathy, bruising, erythema, or edema of the affected joint.

The patient's initial workup was significant for normocytic anemia (hemoglobin of $11.9 \mathrm{~g} / \mathrm{dL}$ and hematocrit of $34.5 \%$ ) and normal liver function tests. She was noted to have elevated inflammatory markers-an erythrocyte sedimentation rate greater than $120 \mathrm{~mm} / \mathrm{h}$ and C-reactive protein of $68.6 \mathrm{mg} / \mathrm{L}$. Urinalysis showed small amounts of leukocyte esterase and bacteria but only $0-5$ white blood cells (WBCs). Urine and blood cultures were obtained and showed no bacterial growth.

An X-ray of the hip showed no acute abnormalities; however, a subsequent MRI showed a small left sacroiliac joint effusion with mild marrow edema that was concerning for infectious or inflammatory sacroiliitis (Figure 1(a)). Scattered small round lesions replaced bone marrow throughout the iliac architecture.

The patient was admitted with a working diagnosis of septic arthritis and received clindamycin intravenously. Joint fluid obtained via CT-guided aspiration revealed clear fluid with 15 WBCs, 200 red blood cells, and sterile cultures. Antibiotics were discontinued given that these findings were inconsistent with pyogenic arthritis, and she was improving clinically. Concern for possible leukemia based on the abnormal bony lesions led to an oncologic evaluation, including a chest X-ray, peripheral blood smear, and measurement of lactate dehydrogenase and uric acid, all of which were within normal limits.

Due to recrudesce of fever following the discontinuation of antibiotics, Epstein-Barr virus, cytomegalovirus, and $B$. henselae antibodies were measured. The patient's fever again declined without directed therapy, and she was discharged home with a diagnosis of transient synovitis.

The patient had intermittent low-grade fevers the week following her discharge. Her $B$. henselae titers were suggestive of recent infection (IgG $>1: 1024$, IgM negative). Due to known hepatic involvement of $B$. henselae in association with disseminated Bartonella disease, imaging (abdominal ultrasound followed by CT scan; Figure 1(b)) was performed and showed multiple liver lesions thought to be consistent with disseminated $B$. henselae infection. Cytopathology of the biopsied sample taken from the hepatic lesions revealed scars consistent with resolving Bartonella liver lesions. While we observed inflammation, we did not observe the granulomatous inflammation that is a more classic sign of CSD. PCR of the lesions was negative for $B$. henselae. Further oncologic evaluations of the hepatic lesions were negative, as was a QuantiFERON-Gold test for tuberculosis.

The patient had clinical resolution after a 6-week azithromycin regimen. An MRI of the patient's pelvis at the end of therapy showed near complete resolution of the abnormalities in the iliac bones, which were fully resolved in an MRI obtained one year later. Furthermore, a CT of the patient's abdomen obtained at the end of therapy revealed that the liver lesions were decreasing in size, consistent with resolving infection. The patient's inflammatory markers also were normal.

\section{Discussion}

CSD often goes unrecognized due to nonspecific signs and symptoms and the disease's usual self-limiting natural course. Osteomyelitis caused by $B$. henselae is rare (observed in $0.1 \%-0.3 \%$ of CSD patients) but has long been recognized to occur in both immune compromised and immune competent patients $[9,10]$. Although PCR is generally considered more sensitive for the diagnosis of $B$. henselae infection than is serology, its sensitivity is still not very good, with a reported range from $43 \%$ to $76 \%$ [4]. Indeed, we were unable to detect $B$. henselae from hepatic sampling via PCR. Insufficient fluid volume precluded testing on the joint sample, and blood was not sampled for PCR detection. The diagnosis of disseminated $B$. henselae was based on significantly elevated titers, radiographic findings, and epidemiologic history.

An oncologic process often is included in the differential diagnoses of patients who present with CSD, as B. henselae can produce osteolytic bone lesions that may be misdiagnosed as tumors $[3,4]$. While an oncologic process was considered in the differential diagnosis of our patient's bony lesions, our patient's symptoms, combined with an unremarkable corroborative diagnostic evaluation and resolution, excluded a malignancy.

Bartonella osteomyelitis most commonly involves the axial skeleton, with several cases reported in the vertebrae, skull, sternum, ribs, and pelvis $[1,11,12]$. While Rozmanic et al. 
documented vertebral and unilateral ilial involvement, our case involves bilateral iliac infection with hepatic lesions and concurrent lack of transaminitis of an immunocompetent child [13]. The pathogenesis of Bartonella osteomyelitis is not well understood. In most cases of Bartonella osteomyelitis, the bone lesion occurs at a distance from the involved lymph nodes, suggesting hematogenous or lymphatic spread of the infection [4, 10, 14]. Bartonella osteomyelitis usually presents subacutely in the axial skeleton, as we observed in our patient, unlike hematogenous osteomyelitis, which presents acutely and typically involves the appendicular skeleton [9].

CSD typically resolves within weeks to months regardless of antimicrobial therapy due to its self-limiting nature. However, studies show that various antibiotics have been used to treat the multitude of clinical manifestations produced by $B$. henselae [1]. A single clinical trial that showed mild-to-moderate hastening of initial recovery with azithromycin therapy supports its use as a first-line agent for lymphadenopathy [15]. However, azithromycin's efficacy in treating patients with atypical CSD, including patients with osteomyelitis and hepatosplenic involvement, has yet to be evaluated [4, 15-17]. Our patient's history of significant cat exposure, elevated anti- $B$. henselae IgG titer, and radiographic findings in bone and liver supported the diagnosis of CSD. We treated our patient with azithromycin given the severity of her symptoms and the evidence of multisystem involvement. After a prolonged course of therapy, she appears to have made a full recovery.

\section{Conflicts of Interest}

The authors declare that there are no conflicts of interest regarding the publication of this article.

\section{References}

[1] K. Puri, A. J. Kreppel, and E. P. Schlaudecker, "Bartonella osteomyelitis of the acetabulum: case report and review of the literature," Vector-Borne and Zoonotic Diseases, vol. 15, no. 8, pp. 463-467, 2015.

[2] J. C. Garcia, M. J. Nunez, B. Castro, J. M. Fernandez, A. Portillo, and J. A. Oteo, "Hepatosplenic cat scratch disease in immunocompetent adults," Medicine, vol. 93, no. 17, pp. 267-279, 2014.

[3] S. Joychan, Y. Kuchipudi, P. J. Danielsky, K. M. Bovid, and G. Deepak, "Case of nonspinal osteomyelitis due to Bartonella and review of the literature," Infectious Diseases in Clinical Practice, vol. 25, no. 5, pp. 240-242, 2017.

[4] K. Mazur-Melewska, K. Jonczyk-Potoczna, A. Mania et al., "The significance of Bartonella henselae bacteria for oncological diagnosis in children," Infectious Agents and Cancer, vol. 10, no. 1, p. 30, 2015.

[5] P. Brouqui and D. Raoult, "Arthropod-borne diseases in homeless," Annals of the New York Academy of Sciences, vol. 1078, no. 1, pp. 223-235, 2006.

[6] S. A. Klotz, V. Ianas, and S. P. Elliot, "Cat-scratch disease," American Family Physician, vol. 83, no. 2, pp. 152-155, 2011.

[7] Cat-scratch disease, 2018, https://www.cdc.gov/healthypets/ diseases/cat-scratch.html.

[8] E. Metzkor-Cotter, Y. Kletter, B. Avidor et al., "Long-term serological analysis and clinical follow-up of patients with cat scratch disease," Clinical Infectious Diseases, vol. 37, no. 9, pp. 1149-1150, 2018.

[9] Y. Kodama, N. Maeno, J. Nishi et al., "Multifocal osteomyelitis due to Bartonella henselae in a child without focal pain," Journal of Infection and Chemotherapy, vol. 13, no. 5, pp. 350-352, 2007.

[10] N. Hajjaji, L. Hocqueloux, R. Kerdraon, and L. Bret, "Bone infection in cat-scratch disease: a review of the literature," Journal of Infection, vol. 54, no. 5, pp. 417-421, 2007.

[11] I. Pons, I. Sanfeliu, N. Cardenosa, M. M. Nogueras, B. Font, and F. Segura, "Serological evidence of Bartonella henselae infection in healthy people in Catalonia, Spain," Epidemiology and Infection, vol. 136, no. 12, pp. 1712-1716, 2008.

[12] S. R. Boggs and R. G. Fisher, "Bone pain and fever in an adolescent and his sibling," Pediatric Infectious Disease Journal, vol. 30, no. 1, p. 89, 2011.

[13] V. Rozmanic, S. Banc, D. Miletic, K. Manestar, S. Kamber, and S. Paparic, "Role of magnetic imaging and scintigraphy in the diagnosis and follow-up of osteomyelitis in cat-scratch disease," Journal of Paediatrics and Child Health, vol. 43, no. 7-8, pp. 568-570, 2007.

[14] M. Al-Rahawan, B. Gray, C. Mitchell, and S. Smith, "Thoracic vertebral osteomyelitis with paraspinous mass and intraspinal extension: an atypical presentation of cat-scratch disease," Pediatric Radiology, vol. 42, no. 1, pp. 116-119, 2012.

[15] J. W. Bass, B. C. Freitas, A. D. Freitas et al., "Prospective randomized double blind placebo-controlled evaluation of azithromycin for treatment of cat-scratch disease," Pediatric Infectious Disease Journal, vol. 17, no. 6, pp. 447-452, 1998.

[16] D. Dornbos, J. Morin, J. R. Watson, and J. Pindrik, “Thoracic osteomyelitis and epidural abscess formation due to cat scratch disease: case report," Journal of Neurosurgery: Pediatrics, vol. 18, no. 6, pp. 713-716, 2016.

[17] D. L. Stevens, A. L. Bisno, H. F. Chambers et al., "Practice guidelines for the diagnosis and management of skin and soft tissue infections: 2014 update by the Infectious Diseases Society of America," Clinical Infectious Diseases, vol. 59, no. 2, pp. e10-e52, 2014. 


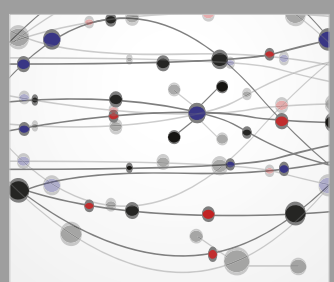

The Scientific World Journal
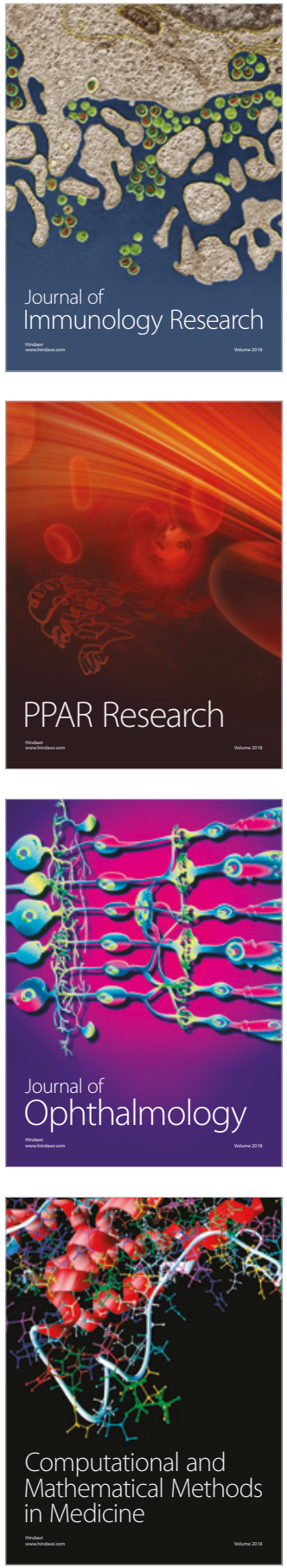

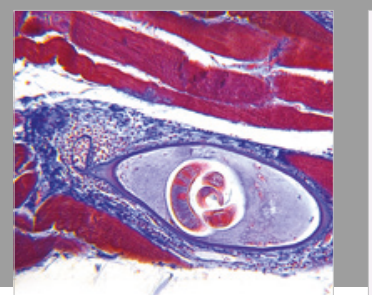

Gastroenterology Research and Practice

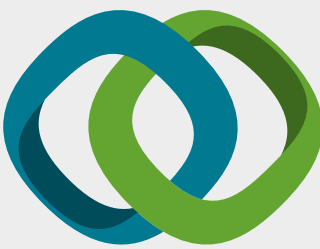

\section{Hindawi}

Submit your manuscripts at

www.hindawi.com
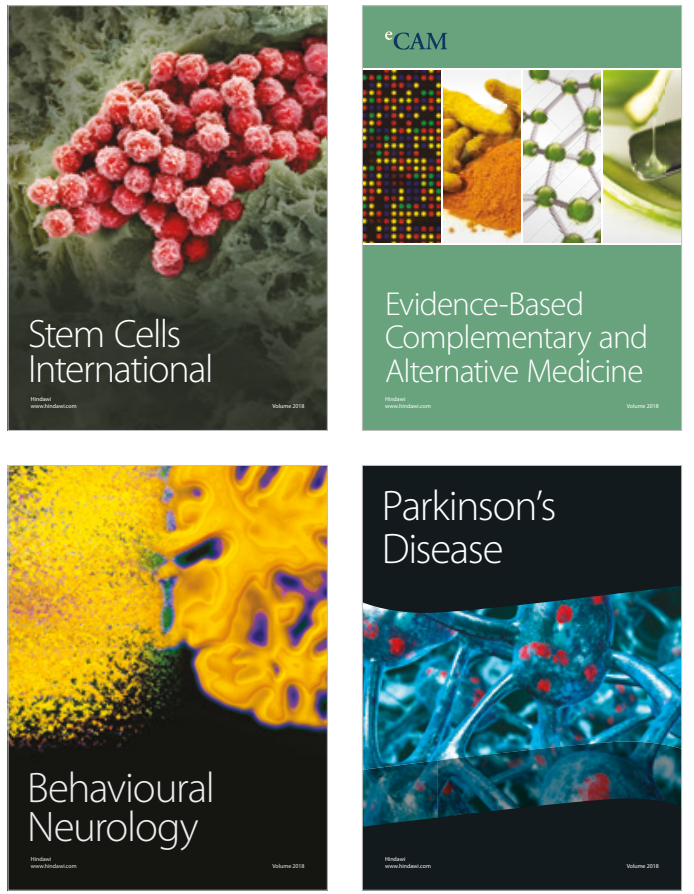

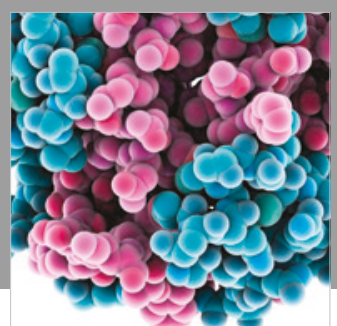

ournal of

Diabetes Research

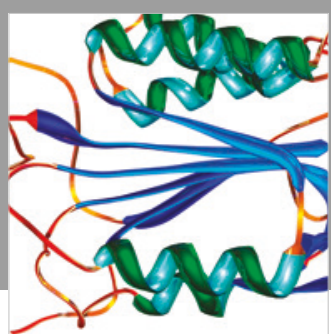

Disease Markers
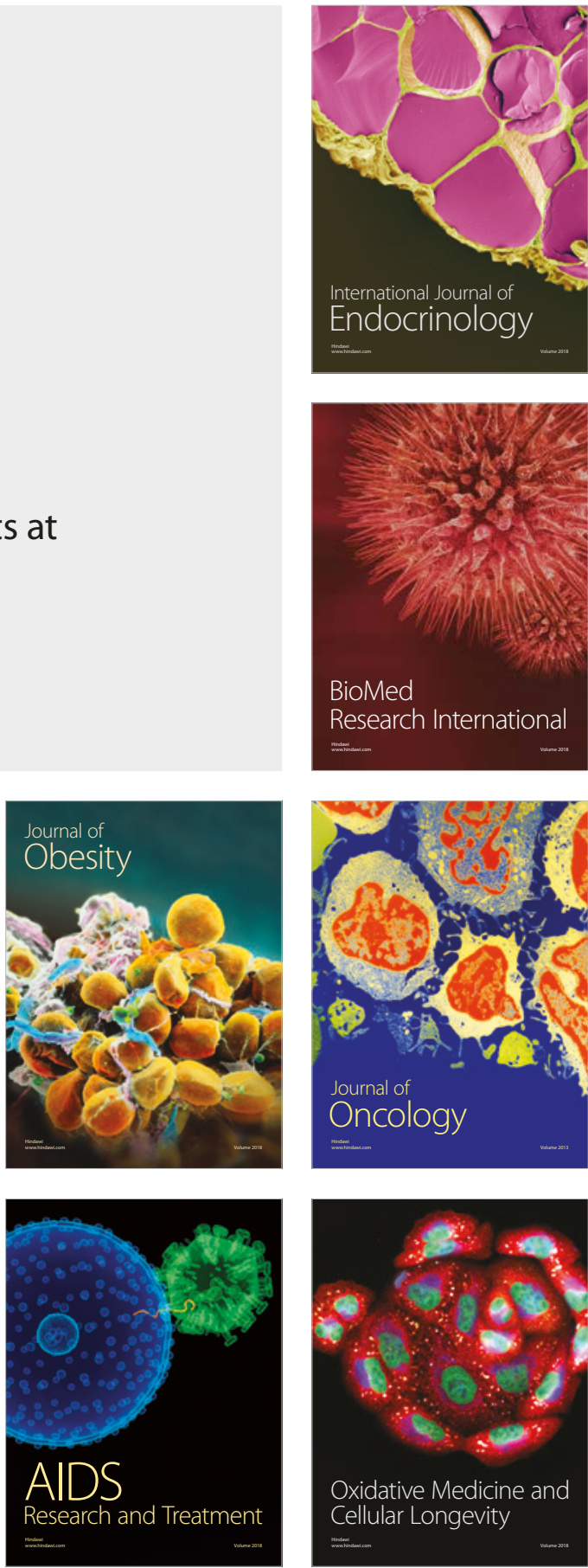